Article

\title{
Structural Features Mediating Zinc Binding and Transfer in the AztABCD Zinc Transporter System
}

\author{
Anusha Meni and Erik T. Yukl *(D) \\ Department of Chemistry and Biochemistry, New Mexico State University, Las Cruces, NM 88003, USA; \\ meni@nmsu.edu \\ * Correspondence: etyukl@nmsu.edu; Tel.: +01-575-646-3176
}

Received: 16 July 2020; Accepted: 4 August 2020; Published: 6 August 2020

\begin{abstract}
Many bacteria require ATP binding cassette $(\mathrm{ABC})$ transporters for the import of the essential metal zinc from limited environments. These systems rely on a periplasmic or cell-surface solute binding protein (SBP) to bind zinc with high affinity and specificity. AztABCD is one such zinc transport system recently identified in a large group of diverse bacterial species. In addition to a classical SBP (AztC), the operon also includes a periplasmic metallochaperone (AztD) shown to transfer zinc directly to AztC. Crystal structures of both proteins from Paracoccus denitrificans have been solved and suggest several structural features on each that may be important for zinc binding and transfer. Here we determine zinc binding affinity, dissociation kinetics, and transfer kinetics for several deletion mutants as well as a crystal structure for one of them. The results indicate specific roles for loop structures on AztC and an N-terminal motif on AztD in zinc binding and transfer. These data are consistent with a structural transfer model proposed previously and provide further mechanistic insight into the processes of zinc binding and transfer.
\end{abstract}

Keywords: zinc; ABC transporter; metal homeostasis; chaperone

\section{Introduction}

Transition metals such as zinc, iron, manganese, and copper provide essential cellular functions, yet are highly toxic in their "free" forms or bound to incorrect targets. Thus, all living things must carefully maintain metal homeostasis. In general, this is accomplished by regulating the uptake and efflux of metals from the cell as well as by proteins that bind, store, and/or shuttle metals to their appropriate destinations. Proteins of the latter description are known as metallochaperones. Well-studied examples include the Atox1, CCS, and Cox17 metallochaperones that deliver copper to specific targets in eukaryotes [1], and the metallothioneins that buffer zinc concentration in eukaryotes [2] and a few bacteria [3]. A new family of intracellular zinc-binding GTPases called COG0523 has been identified in bacteria. These are often upregulated by zinc starvation [4-6] and may function as metallochaperones. However, direct evidence of metal transfer to target proteins has never been demonstrated, and their physiological functions are often unclear.

Metal binding proteins outside the cell are also important for bacterial metal homeostasis. ATP binding cassette (ABC) transporters are critical for high affinity uptake of transition metals and other nutrients. In addition to dimeric membrane permease and cytosolic ATPase proteins, bacterial ABC transporters rely on periplasmic (Gram-negative) or cell surface (Gram-positive) solute binding proteins (SBP) $[7,8]$. The SBP is essential to bind the substrate with high affinity and specificity and deliver it to the membrane permease for transport into the cell. SBPs have been categorized according to structure and substrate specificity [9], with members of group A-I mediating transport of 
zinc, manganese, or iron. The presence of a flexible loop rich in His/Asp/Glu residues near the metal binding site is indicative of zinc specificity [10]. These proteins have garnered considerable interest as potential antibiotic targets as they are required for high affinity zinc import and survival under the extremely zinc-limited conditions imposed by animal hosts [11,12].

The cluster A-I SBPs are not the only extracellular bacterial zinc metallochaperones. In a surprising number of cases, metal may be transferred to them by other metallochaperones. Examples of such chaperones include ZinT [13-19], the polyhistidine triad protein PhtD [20-22], and AztD [23]. Direct zinc transfer to an SBP has only been demonstrated for the latter two, and the mechanistic details of this process are unclear. This is typically the case despite the importance of zinc transfer processes in biology due to the lack of a convenient spectroscopic handle for this element. However, the kinetics of transfer from AztD to AztC can be easily followed by a roughly 2-fold increase in intrinsic protein fluorescence of AztC upon zinc binding [24]. This combined with crystal structures of both AztC [24,25] and AztD [26] make this system ideal for detailed mechanistic investigations of direct, protein-to-protein metal transfer as well as zinc binding/dissociation from solution.

Our previous work culminated in a docking model showing the possible transfer complex formed between AztC and AztD (Figure 1). This model highlights several important structural features. First, the flexible loop of AztC that we refer to as the D-Loop projects into the central pore of the AztD beta-propeller structure. This loop and each of its 3 His residues are essential for transfer [25]. Secondly, AztC (residues 222-229) referred to as the Z-loop must be displaced away from the zinc binding site in order for the complex to form. This is true in the apo AztC structure, but this loop closes down over the zinc site in the holo structure. This was suggested to provide a means for dissociation of the complex after transfer. Finally, the $6 \mathrm{~N}$-terminal residues after cleavage of the periplasmic targeting sequence of AztD (residues 22-27) are not observed in the crystal structure, but would be positioned at the interface with AztC according to the model. Residues 23-29 form a DHDHDHE motif that may bind zinc and/or facilitate transfer to AztC. Zinc binding to this N-terminal motif (NTM) was suggested by the observation of a third, relatively low-affinity binding site in addition to the two high-affinity sites in AztD [23].

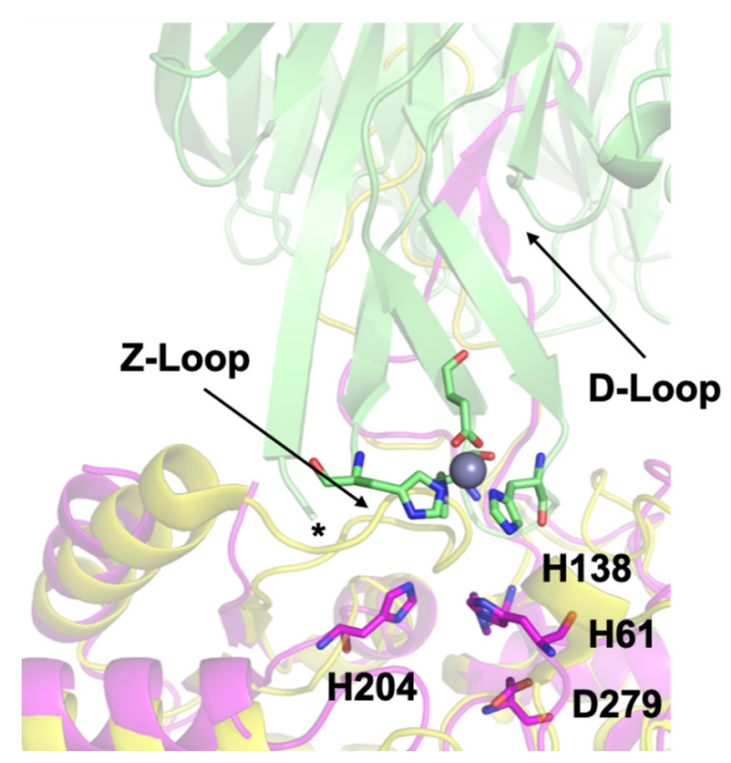

Figure 1. Docking model of the AztC-AztD transfer complex. AztD is shown in green with zinc binding residues shown as sticks colored according to element and zinc as a gray sphere. The first modeled $\mathrm{N}$-terminal residue of AztD (His 28) is indicated by an asterisk. Apo AztC is shown in magenta with zinc binding residues labeled and shown as sticks colored according to element. Holo AztC superimposed on the apo form is shown in yellow, illustrating how the position of the Z-loop in this structure would block zinc transfer. 
Here we utilize deletion mutants and the convenient fluorescence behavior of AztC to characterize the role of each of the above structural elements on zinc dissociation from AztC and transfer from AztD. Each appears to have a unique effect on either or both processes that is consistent with the proposed model. Further, we sought to generate a mutant of AztC that would be capable of binding to AztD but unable to accept zinc from it. We reasoned that such a mutant would act as a competitive inhibitor of zinc transfer and would provide a means for determining the affinity between the two proteins. Thus, we generated an AztC mutant lacking the two zinc ligands His 138 and His 204 (Figure 1) that we refer to as H138/204A, and we evaluated its ability to competitively inhibit transfer to WT AztC. Remarkably, the presence of this mutant actually enhanced the rate of transfer from AztD. This last intriguing finding suggests additional complexity to the transfer event and provides avenues for further study of this important process.

\section{Materials and Methods}

\subsection{Expression and Purification of Proteins}

WT and mutant AztC [24] and AztD [23] were expressed and purified as previously described. All mutants were generated using the Q $5^{\circledR}$ Site-Directed Mutagenesis Kit (New England BioLabs, Ipswich, MA, USA) and confirmed by plasmid sequencing. Presence and purity of all proteins were confirmed using SDS-PAGE, and concentration was measured using extinction coefficients determined by the method of Edelhoch [27]. Apo proteins were generated as previously by dialysis at $4{ }^{\circ} \mathrm{C}$ against $50 \mathrm{mM} \mathrm{NaOAc}$ buffer $\mathrm{pH}$ 4.5, $50 \mathrm{mM}$ EDTA, and $150 \mathrm{mM} \mathrm{NaCl}$ followed by dialysis against $50 \mathrm{mM}$ tris buffer pH 8.0, $150 \mathrm{mM} \mathrm{NaCl}$, and $3.4 \mathrm{~g} / \mathrm{L}$ Chelex ${ }^{\circledR}$ (Bio-Rad, Hercules, CA, USA).

\subsection{Mag-Fura 2 Competition Assay}

Zinc binding affinities were measured using a Mag-Fura 2 (MF2, Invitrogen, Carlsbad, CA, USA) competition assay derived from Golynskiy et al. [28] as previously described [23,24]. All fluorescence measurements were made using a Varian Cary Eclipse (Palo Alto, CA, USA) fluorescence spectrophotometer with entrance and exit slits set to $10 \mathrm{~nm}$. Protein concentration was measured before each experiment and MF2 concentration was determined using an extinction coefficient at $369 \mathrm{~nm}$ of 22,000 $\mathrm{M}^{-1} \times \mathrm{cm}^{-1}$ [28]. In each experiment, $1.0 \mu \mathrm{M}$ apo protein and $0.5 \mu \mathrm{M}$ MF2 in binding buffer (20 mM HEPES pH 7.4, $200 \mathrm{mM} \mathrm{NaCl}, 5 \%$ v/v glycerol treated with Chelex ${ }^{\circledR}$ resin) were titrated with increasing concentrations of $\mathrm{ZnCl}_{2}$, keeping the total volume of titrant added to less than $10 \% v / v$. Fluorescence excitation spectra were scanned from $250-450 \mathrm{~nm}$ while monitoring emission at $505 \mathrm{~nm}$. Experiments were performed in triplicate and the fluorescence intensities at $\lambda_{\mathrm{ex}}=330 \mathrm{~nm}$ were fit using the program DYNAFIT v. 4.05.103 [29,30] using scripts adapted from Golynskiy et al. [28]. Prior to each series of experiments, the affinity of MF2 for zinc in our buffer system was determined using DYNAFIT and used in our calculation of protein binding affinity.

\subsection{Zinc Dissociation}

Apo WT or mutant AztC was diluted to $1 \mu \mathrm{M}$ in binding buffer in a stirred cuvette at ambient temperature. Protein was titrated with up to 3 equivalents of $\mathrm{ZnCl}_{2}$ and the fluorescence intensity monitored to ensure saturation. Dissociation was initiated by the addition of EDTA to $1.0 \mathrm{mM}$ and the decay in emission intensity at $315 \mathrm{~nm}$ was monitored. Data was fit by a single exponential function using DYNAFIT $[29,30]$.

\subsection{Zinc Binding and Transfer by Intrinsic Fluorescence}

All fluorescence measurements were made using a Varian Cary Eclipse fluorescence spectrophotometer as previously described. Titration experiments were performed with AztC proteins at $10 \mu \mathrm{M}$ in binding buffer. These were titrated with $\mathrm{ZnCl}_{2}$ or AztD reconstituted with 2 equivalents of $\mathrm{ZnCl}_{2}$ followed by desalting into binding buffer using $\mathrm{Zeba}^{\mathrm{TM}}$ spin desalting columns 
(Pierce Biotechnology ${ }^{\mathrm{TM}}$, Rockford, IL, USA). In titrations of apo-AztC with holo-AztD, 5-15 min of equilibration was allowed between measurements. After addition of holo-AztD to $14 \mu \mathrm{M}, 20 \mu \mathrm{M}$ of $\mathrm{ZnCl}_{2}$ was added to determine AztC saturation.

Kinetic experiments were performed as previously described [26] with minor modifications. Apo AztC at $0.5 \mu \mathrm{M}$ in binding buffer containing $1 \mathrm{mM}$ EDTA was placed in a stirred cell at ambient temperature. Transfer was initiated by addition of varying concentrations of AztD reconstituted as above but omitting the desalting step. Fluorescence emission at $315 \mathrm{~nm}\left(\lambda_{\text {exc }}=278 \mathrm{~nm}\right)$ was recorded over time. Slit widths were varied to optimize signal to noise and avoid saturation at high protein concentration. First order fits to the data were generated using the onboard Cary Eclipse software. Inhibition experiments were performed similarly to those above but included varying concentrations of H138/204A or $\Delta \mathrm{D}$-loop AztC in addition to WT AztC at $0.5 \mu \mathrm{M}$. Transfer was initiated by addition of $0.5 \mu \mathrm{M}$ reconstituted WT AztD and monitored as above.

\subsection{Crystallization and Structure Determination}

$\Delta$ Z-loop AztC were crystallized under the same conditions used for holo WT [24]. Crystals were cryoprotected with mother liquor containing $10 \%$ glycerol prior to cryocooling in liquid nitrogen. Diffraction data was collected at $100 \mathrm{~K}$ on beamline 5.0.2 at the Advanced Light Source at Berkeley National Laboratory and indexed, integrated, and scaled with XDS v. 0.92 [31,32]. The WT AztC structure (PDB ID: 5W57) [24] was used as the search model for molecular replacement using Phaser-MR [33] module of Phenix v. 1.14-3260. Manual model building was done in Coot v. 0.7 [34] and further rounds refinement were done using Phenix Refine [35]. Coordinates of $\Delta Z$-loop AztC have been deposited in the PDB with entry code 6XPN. Figures were prepared using Pymol v. 2.1, which was also used for pairwise structural alignments.

\section{Results}

\subsection{Zinc Binding and Dissociation}

The zinc binding affinity of WT AztD, WT AztC, $\Delta \mathrm{D}$-Loop AztC, and $\Delta \mathrm{Z}$-Loop AztC were previously determined by competition assay with the fluorophore MagFura-2 (MF2) (Table 1). Here we apply this technique to H138/204A AztC and a deletion of the N-terminal motif (residues 23-29) in AztD ( $\triangle$ NTM AztD) (Figure 2). The former binds approximately one equivalent (0.85) of zinc with a $\mathrm{Kd}$ below the detection limit of this assay $(\leq 0.1 \mathrm{nM})$, making it indistinguishable from WT. $\triangle \mathrm{NTM}$ AztD binds two equivalents of zinc with very high affinity. Previous work with WT AztD indicated some negative cooperativity between high affinity binding sites. Intriguingly, this appears to be largely eliminated in the $\triangle$ NTM mutant. Further, the third binding site in WT AztD is not observed, consistent with relatively low affinity zinc binding by this motif. We also evaluated zinc binding to H138/204A by intrinsic protein fluorescence (Figure 2C,D). Like WT, this mutant exhibits a roughly 2-fold increase in fluorescence emission intensity saturating at one equivalent of added zinc consistent with high affinity binding.

Table 1. Zinc binding affinity and stoichiometry of WT and mutant AztD.

\begin{tabular}{|c|c|c|c|c|}
\hline Protein & Site & $K_{d} \pm$ S.D. $(n M)$ & $\mathrm{k}_{\mathrm{off}} \pm$ S.D. $\left(\mathrm{min}^{-1}\right)$ & $\mathrm{k}_{\mathrm{T}}\left(\mathrm{M}^{-1} \times \mathrm{s}^{-1}\right)$ \\
\hline \multirow{3}{*}{ WT AztD [23] } & 1 & $0.7 \pm 0.3$ & & \\
\hline & 2 & $54 \pm 8$ & & \\
\hline & 3 & $340 \pm 110$ & & \\
\hline \multirow{2}{*}{$\Delta \mathrm{S} 1 \mathrm{AztD}[26]$} & 2 & $1.3 \pm 0.7$ & & \\
\hline & 3 & $248 \pm 81$ & & \\
\hline \multirow{2}{*}{$\Delta S 2$ AztD [26] } & 1 & $0.1^{*}$ & & \\
\hline & 3 & $158 \pm 35$ & & \\
\hline
\end{tabular}


Table 1. Cont.

\begin{tabular}{|c|c|c|c|c|}
\hline Protein & Site & $K_{d} \pm$ S.D. $(n M)$ & $k_{\text {off }} \pm$ S.D. $\left(\min ^{-1}\right)$ & $\mathrm{k}_{\mathrm{T}}\left(\mathrm{M}^{-1} \times \mathrm{s}^{-1}\right)$ \\
\hline \multirow{2}{*}{$\Delta \mathrm{NTM}$ AztD } & 1 & $0.1^{*}$ & & \multirow{2}{*}{$1.50 \times 10^{-3}$} \\
\hline & 2 & $0.2 \pm 0.1$ & & \\
\hline WT AztC [24] & 1 & $0.3 \pm 0.1$ & $1.2 \pm 0.1 \times 10^{-3}$ & $\begin{array}{c}1.33 \times 10^{-3}[26] \\
1.55 \times 10^{-3} \text { (this work) }\end{array}$ \\
\hline$\Delta$ D-Loop AztC [25] & 1 & $0.2 \pm 0.1$ & $0.9 \pm 0.1 \times 10^{-3}$ & nd \\
\hline$\Delta$ Z-Loop AztC [25] & 1 & $0.2 *$ & $7.0 \pm 0.1 \times 10^{-3}$ & $2.92 \times 10^{-3}$ \\
\hline H138/204A AztC [24] & 1 & $0.1 *$ & $623.4 \pm 0.5 \times 10^{-3}$ & \\
\hline
\end{tabular}

* Uncertainties for this value could not be estimated as the Kd appears to be below the detection limit of this assay. The data was fitted with the indicated value.
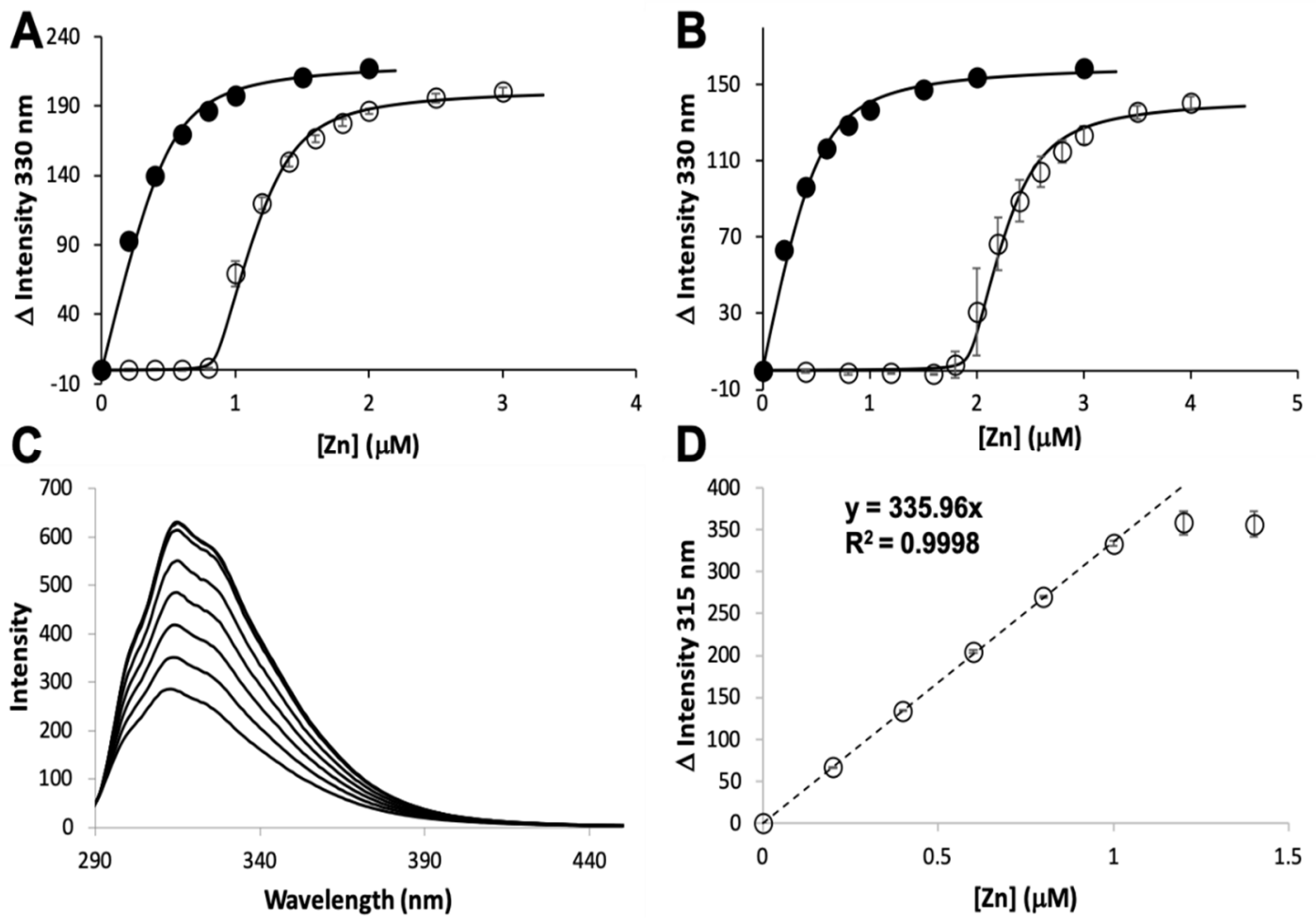

Figure 2. Zinc binding by AztC and AztD mutants. Intensity change of MF2 at $330 \mathrm{~nm}$ with increasing zinc in the absence (solid circles) and presence (empty circles) of apo H138/204A AztC (A) and $\Delta$ N-terminal motif (NTM) AztD (B). (C) Fluorescence emission spectra of H138/204A AztC $\left(\lambda_{\text {exc }}=278 \mathrm{~nm}\right)$ and (D) magnitude of the fluorescence intensity change with increasing zinc concentration. Titrations containing protein were performed in triplicate and error bars represent the standard deviation between measurements. Least squared fits are shown as solid lines for (A) and (B) and a dotted line for the linear fit in (D) with slope and $R^{2}$ values indicated.

The MF2 assay does not reveal any significant differences between the affinities of the various AztC mutants, likely because each $\mathrm{Kd}$ is near or below the detection limit. Thus, we developed an intrinsic fluorescence assay to monitor the zinc off-rate for each (Table 1). Briefly, protein is saturated with zinc as determined by fluorescence emission (Figure 3A). Each of the AztC proteins investigated here is similar in terms of the magnitude of fluorescence intensity change upon zinc binding. Next, a large excess of EDTA is added to capture metal as it dissociates, and the decrease in fluorescence intensity is monitored over time as a direct measure of zinc loss. As expected, the zinc off-rates for WT and $\Delta \mathrm{D}$-Loop AztC are comparable and extremely slow (Figure 3B,C). However, deletion of the Z-loop causes a more than 5-fold increase in off-rate (Figure 3D), consistent with the function of this feature in closing down over the zinc site. Mutation of the two zinc ligands His138 and His204 causes a dramatic 
increase in off-rate, approximately 600 times faster than WT (Figure 3E). Assuming an upper limit to the $\mathrm{Kd}$ of $0.1 \mathrm{nM}$ as indicated from the MF2 assay, this suggests that zinc binding to this mutant is near the diffusion limit, around $10^{8} \mathrm{M}^{-1} \times \mathrm{s}^{-1}$.
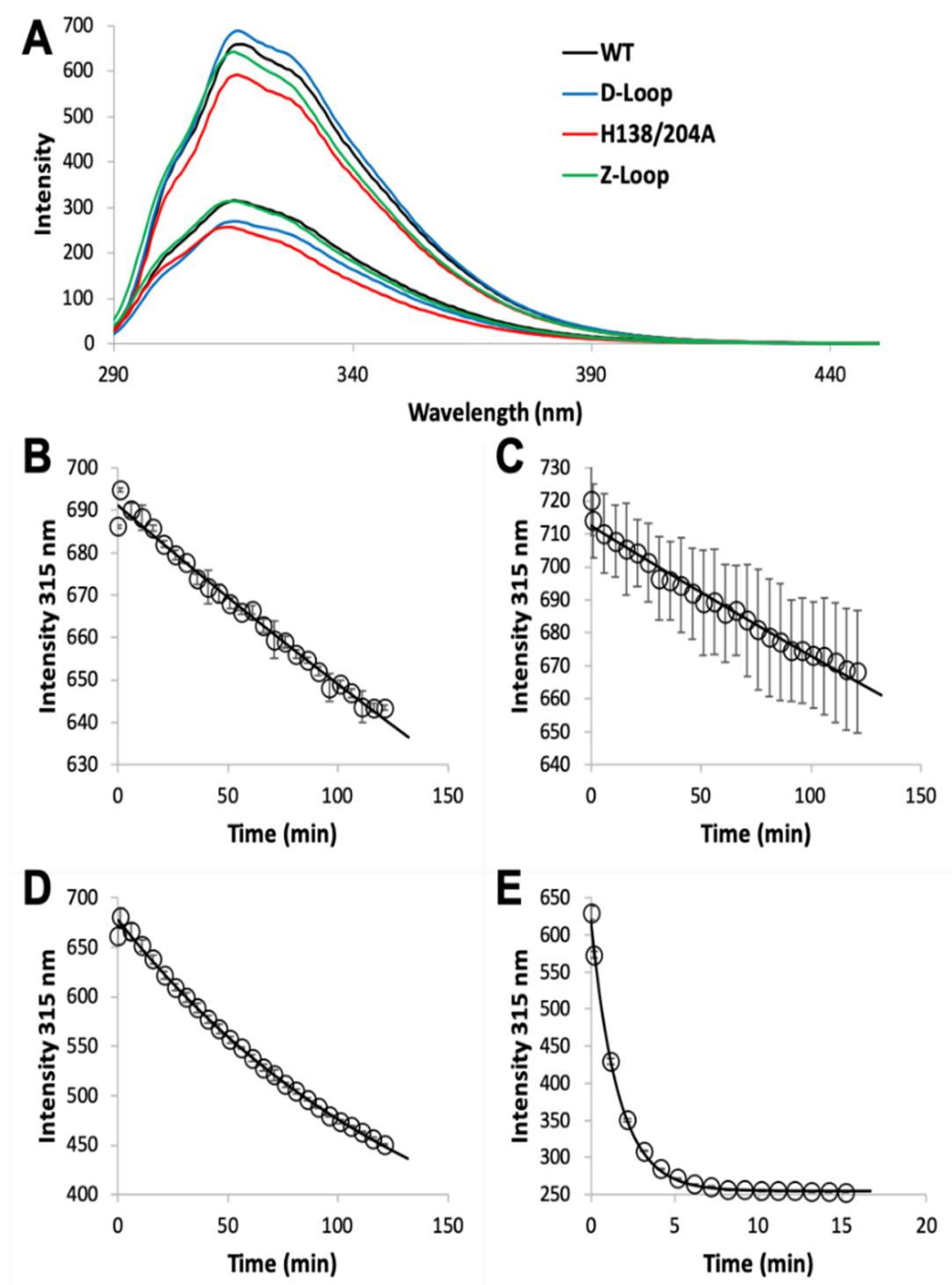

Figure 3. Zinc dissociation from WT and mutant AztC. (A) Fluorescence emission spectra of WT (black), $\Delta$ D-Loop (blue), $\Delta$ Z-Loop (green) and H138/204A (red) AztC before and after addition of saturating $\mathrm{ZnCl}_{2}$. Upon addition of excess EDTA, the emission intensity at $315 \mathrm{~nm}$ was tracked over time for WT (B), $\Delta$ D-Loop (C), $\Delta$ Z-Loop (D), and H138/204A (E) AztC. Dissociation experiments were performed in triplicate and error bars represent the standard deviation between measurements.

\subsection{Zinc Transfer from AztD}

Transfer of zinc from AztD to AztC can be followed by the increase in AztC fluorescence upon zinc binding $[23,25,26]$. Previous work indicates that this process requires the presence of the D-Loop, but transfer was still observed to $\Delta$ Z-Loop AztC. However, the kinetics were not previously evaluated. Here we demonstrate that the rate of transfer from WT AztD to $\triangle \mathrm{Z}$-Loop AztC is increased approximately two-fold relative to WT AztC under the same conditions (Table 1, Figure 4). In contrast, deletion of the NTM from AztD appears to have no effect on the rate of transfer to WT AztC. 

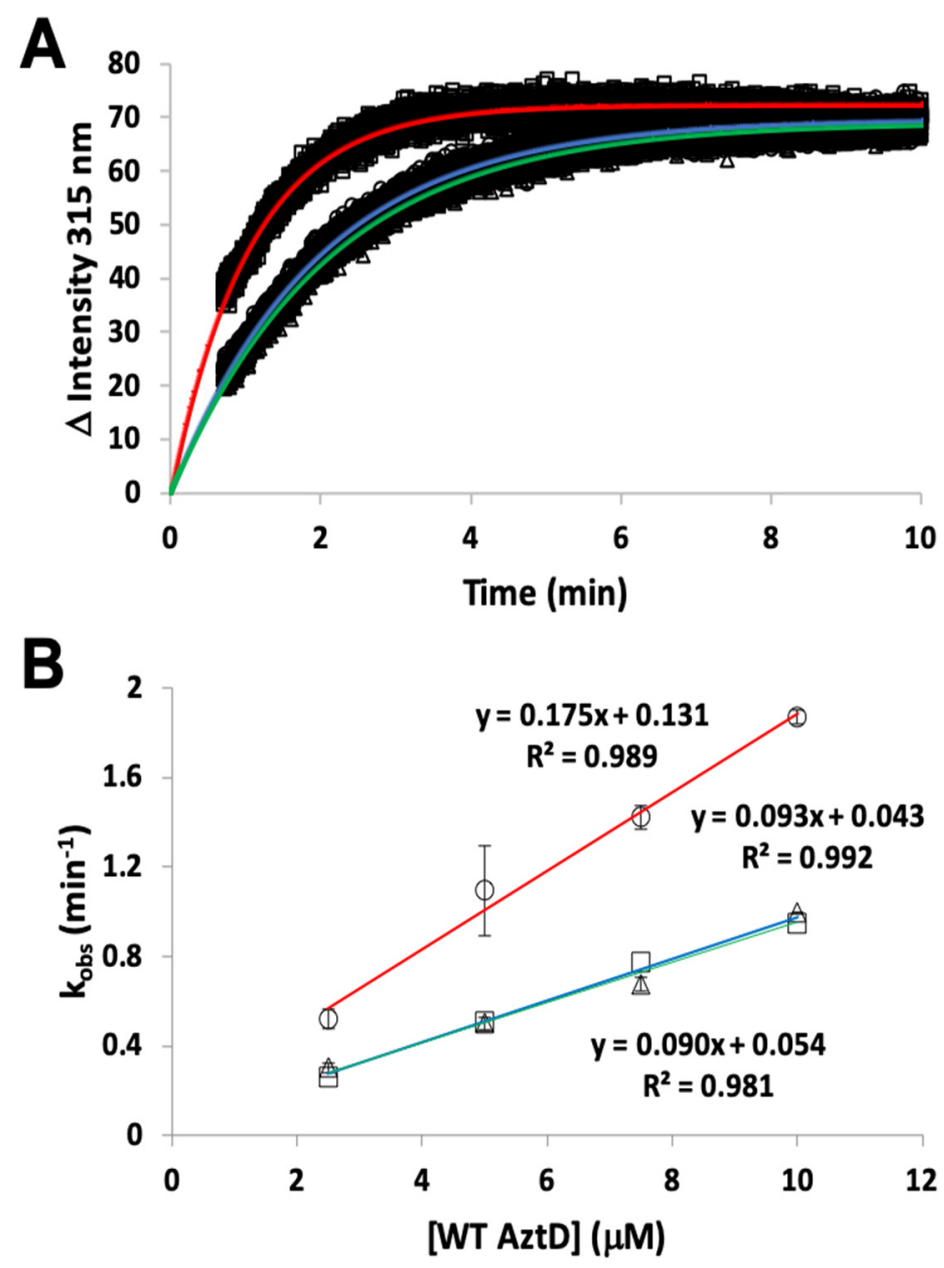

Figure 4. Kinetics of zinc transfer between mutants of AztD and AztC. (A) Representative kinetic data from standard fluorescence emission experiments for WT AztD to WT AztC (circles), $\triangle N T M$ AztD to WT AztC (triangles), and WT AztD to $\Delta$ Z-Loop (squares) with fits to a first order kinetic scheme (blue, green and red, respectively). (B) Observed first order rate constant versus AztD concentration for standard fluorescence data under pseudo-first order conditions. Error bars represent standard deviations from duplicate experiments $(n=2)$ except for the WT AztD to WT AztC experiment, which was run a single time to confirm previous data under current conditions. Symbols and linear fit colors are as for $(\mathbf{A})$.

The rate of transfer to H138/204A AztC could not be evaluated because of the rapid off-rate of zinc for this mutant. To determine if zinc transfer to this mutant from AztD was possible, it was titrated with holo AztD in the absence of EDTA (Figure 5). Although the fluorescence changes were smaller than for WT, they were nevertheless greater than for $\Delta \mathrm{D}$-Loop AztC. These changes also did not reach saturation after 1.4 equivalents of AztD were added and appear to have a roughly hyperbolic rather than linear dependence on AztD concentration. Cumulatively, these data suggest that zinc can be transferred to $\mathrm{H} 138 / 204 \mathrm{~A}$ AztC, but the rapid off-rate from this mutant allows an equilibrium to be established between zinc binding to it and AztD. This is in contrast to the virtually unidirectional transfer of zinc to WT AztC, which serves as a kinetic trap for zinc. This also differs from the behavior of $\Delta \mathrm{D}$-Loop AztC, which cannot access zinc from AztD at all. 

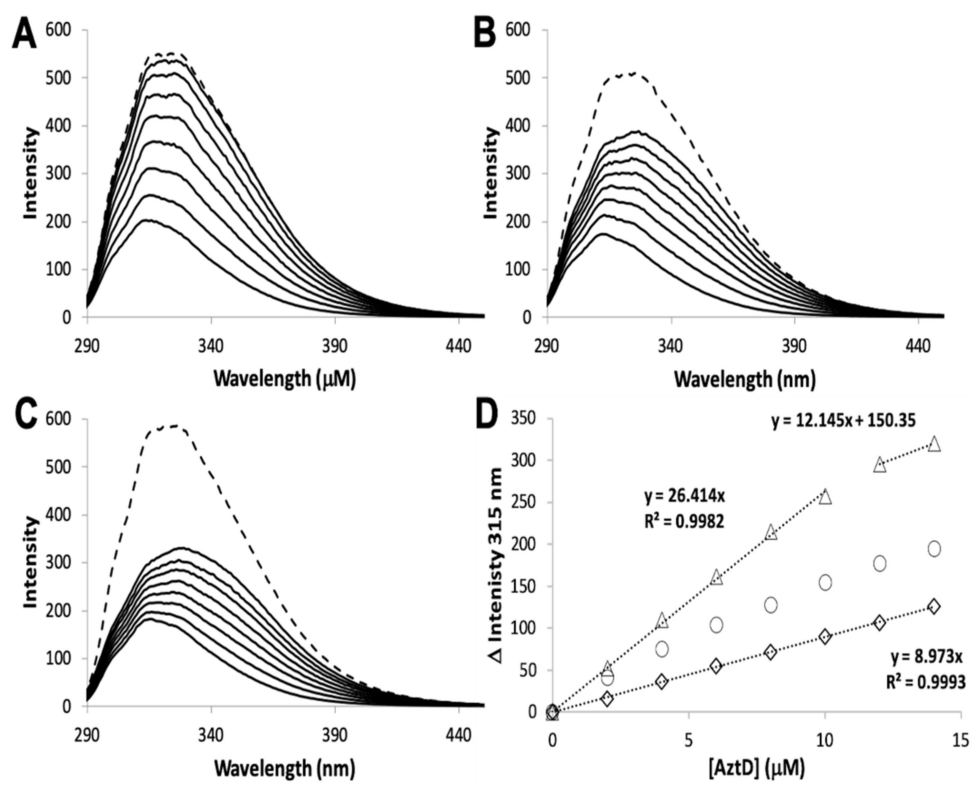

Figure 5. Zinc transfer by intrinsic fluorescence. Reconstituted WT AztD was titrated into apo WT (A), H138/204A (B), or $\Delta$ D-Loop (C) AztC. Fluorescence emission spectra $\left(\lambda_{\text {exc }}=278 \mathrm{~nm}\right)$ were recorded after each addition of AztD and the intensity change at $315 \mathrm{~nm}$ plotted as a function of AztD concentration (D) with equations for linear fits indicated where appropriate. A saturating concentration of $\mathrm{ZnCl}_{2}$ $(1.1 \mathrm{mM})$ was added after the titration to assess whether transfer from AztD was complete (dotted line, A, B, and C).

\subsection{Crystal Structure of $\Delta Z$-Loop}

In order to determine what structural features of $\Delta$ Z-Loop AztC might mediate its more rapid transfer kinetics, a crystal structure was determined for this mutant (Table 2, Figure 6). The overall structure is very similar to that of the holo WT, with rmsd $=0.26-0.30 \AA$ across all atoms depending on which chains are aligned. The zinc binding site is also completely superimposable. Residues 117-133 (the D-Loop) could not be modeled into either chain of the $\Delta$ Z-Loop AztC structure due to a lack of electron density. Residues 222-229 (the Z-Loop) are absent, leaving a run of four Ala residues. Electron density in this region is also somewhat weak, indicating some flexibility. The deletion shortens the loop between helices $\alpha 7$ and $\alpha 8$, preventing closure of the loop over the zinc binding site as is observed for the WT. The lack of any other significant structure changes as a result of the deletion suggest that this is the primary reason for the increase in zinc dissociation rate and transfer rate from AztD. 
Table 2. X-ray diffraction data collection, processing and refinement statistics.

\begin{tabular}{cc}
\hline & Holo $\Delta$ Z-Loop AztC \\
\hline Data collection & \\
Space group & $\mathrm{P} 2_{1}$ \\
Unit cell parameters & $62.3,105.0,64.3$ \\
a, b, c $(\AA)$ & $90.0,110.8,90.0$ \\
$\alpha, \beta, \gamma\left({ }^{\circ}\right)$ & $46.6-2.26$ \\
Resolution range $(\AA)$ & $133,590 / 36,075$ \\
Number of reflections (measured/unique) & $0.04(0.59)$ \\
Rmerge $_{\text {I }}$ & $18.5(2.3)$ \\
Completeness $(\%)$ & $99.5(99.9)$ \\
Redundancy & $3.7(3.7)$ \\
Refinement Statistics & \\
Resolution $(\AA)$ & $39.5-2.26$ \\
$R_{\text {work }} / R_{\text {free }}$ & $17.4 / 20.8$ \\
Number of atoms & 3,840 \\
Protein & 2 \\
Zinc & 57 \\
Water & 0 \\
Other & \\
R.m.s. deviations & 0.009 \\
Bond lengths $(\AA)$ & 1.20 \\
Bond angles $\left({ }^{\circ}\right)$ & \\
Ramachandran Statistics & $99.5 \%$ \\
Allowed & $0.5 \%$ \\
Outliers & 58.0 \\
Average B-factor $\left(\AA^{2}\right)$ &
\end{tabular}



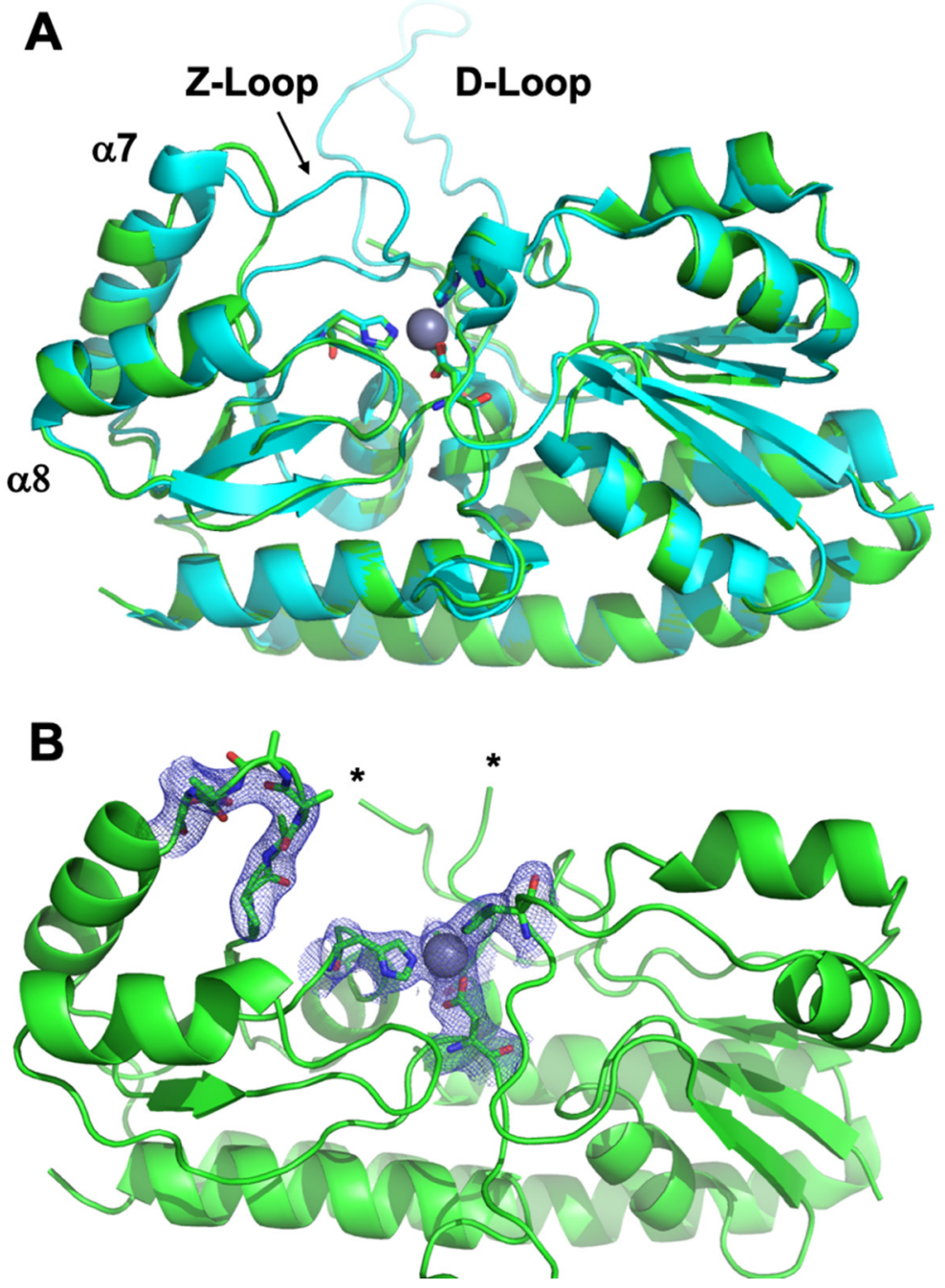

Figure 6. Crystal structure of $\Delta$ Z-Loop AztC. (A) Overlay of $\Delta$ Z-Loop (green) and WT (blue) AztC. Zinc binding residues are shown as sticks colored according to element and zinc is shown as a gray sphere. (B) $\Delta$ Z-Loop AztC showing 2Fo-Fc electron density contoured at 1.0 $\sigma$ for the truncated loop and zinc binding site. Asterisks indicate residues 116 and 134 between which residues could not be modeled due to a lack of electron density.

\subsection{Competition Between AztC Mutants}

We next investigated whether H138/204A AztC might act as a competitive inhibitor for the interaction between WT AztC and AztD. We reasoned that increasing concentrations of H138/204A AztC would slow the rate of transfer to WT AztC as this mutant can interact with AztD but would not show net transfer due to its rapid off-rate. In contrast, we actually observed a dramatic increase in the rate of transfer to WT AztC (Figure 7). To confirm that this was not a result of molecular crowding, we repeated the experiment with increasing concentrations of $\triangle \mathrm{D}$-Loop AztC, which had no impact on transfer to WT AztC. 


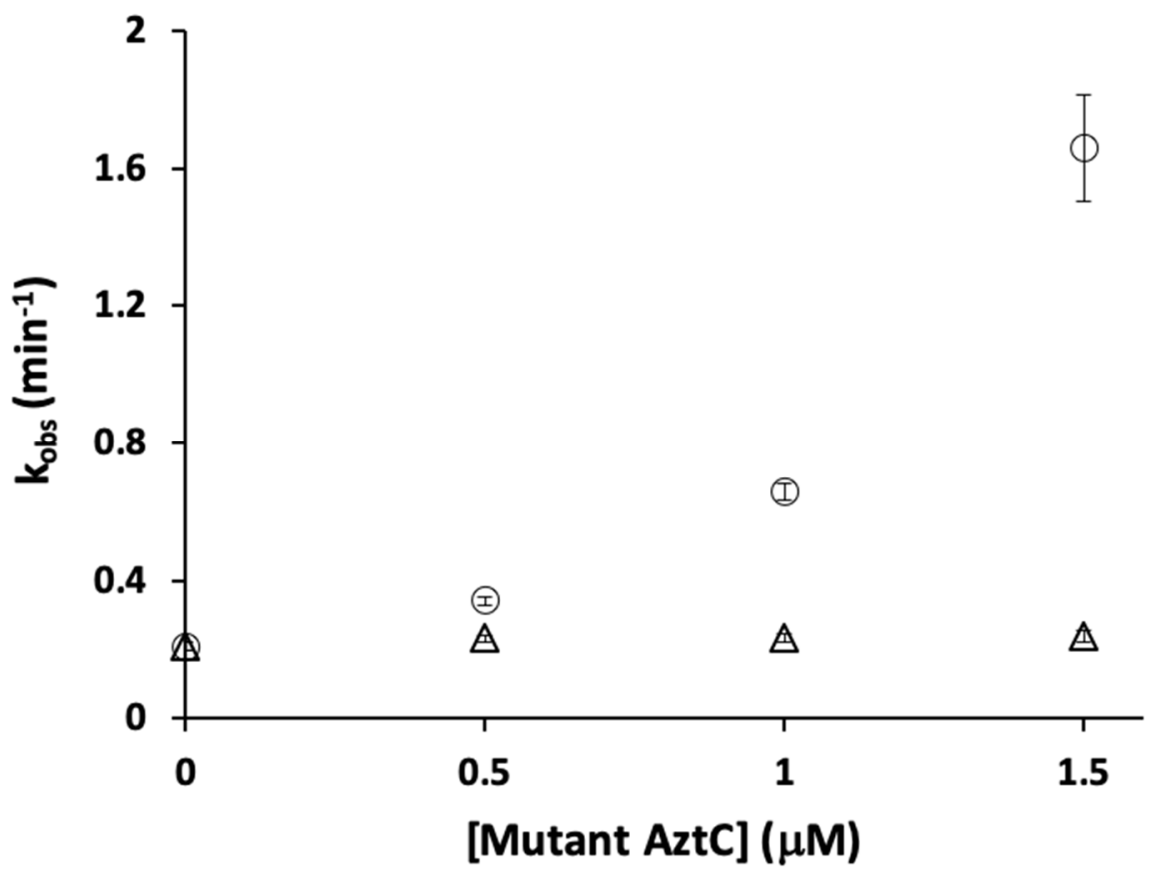

Figure 7. Kinetics of zinc transfer from WT AztD to WT AztC in the presence of mutant AztC. Observed first order rate constant versus H138/204A (circles) or $\Delta \mathrm{D}$-Loop (triangles) AztC concentration. WT AztC and AztD were both present at $0.5 \mu \mathrm{M}$, EDTA was present at $1 \mathrm{mM}$. Error bars represent standard deviations from triplicate experiments $(n=3)$.

\section{Discussion}

The AztC/D system provides an ideal system to study the mechanisms of zinc binding and direct, protein-protein zinc transfer. Here we have systematically evaluated the role of several structural features of AztC and AztD in these processes. We will discuss the impact of each feature individually.

\subsection{The AztD N-terminal Motif (NTM)}

Some AztD homologues contain motifs rich in His/Asp/Glu residues at either termini or occasionally within the interior of the sequence. For example, the other structurally characterized AztD from Citrobacter koseri contains the C-terminal sequence EHHDHEAHHHDDHAH (residues 407-421), which was also absent in that structure due to a lack of electron density [26]. Despite the fact that the termini are situated close to the zinc transfer site in AztD, the NTM appears to play no role in zinc transfer to AztC. However, zinc binding affinity is somewhat altered, with a loss of the third binding site and an apparent loss of negative cooperativity between sites 1 and 2 . It is worth noting that an interaction was observed between sites 1 and 2 of adjacent protomers in the crystal structure of $C$. koseri AztD. The fact that we have never observed AztD dimerization in solution initially suggested that this was simply a crystal packing artifact. However, a weak, transient interaction would explain how these sites might communicate and how deletion of the NTM might eliminate that communication.

\subsection{The AztC Z-Loop}

In the holo structure of AztC, the Z-loop is closed over the zinc site with the carbonyl oxygen of Val 224 a distance of $3.6 \AA$ from the zinc ion. While too far to be considered a coordinate bond, this still likely represents an electrostatic interaction. In the apo AztC structure, the loop is pulled away from the zinc site in one chain and absent in the other due to a lack of electron density. This suggests that the Z-loop is highly flexible in apo AztC and that its position is stabilized in the holo form by the interaction between Val 224 and zinc. 
The Z-loop is unusual in cluster A-I SBP structures. To our knowledge, it has only been observed in the putative manganese transporter TroA [36,37] and the zinc/laminin binding proteins Lmb [38] and Lbp [39]. In TroA, the zinc-Val interaction is conserved at 3.7-3.9 A while in Lmb and Lbp the interaction is between the carbonyl oxygen atom of an Ile residue and zinc at 3.4-3.7 $\AA$. Unlike AztC, the apo form of TroA does not show any significant displacement of the Z-Loop relative to the holo form [40]. Although no apo structure exists for Lmb or Lbp, a recent molecular dynamics simulation of both Lmb and TroA indicated significant flexibility of the Z-Loop in the apo form and displacement away from the zinc binding site upon zinc loss [41].

Deletion of the Z-Loop in AztC causes a roughly 6-fold increase in the dissociation rate of zinc relative to $\mathrm{WT}$, suggesting that closure of this loop over the zinc slows its dissociation. The rate of transfer from AztD also increased. The loop is flexible in the apo form and may sample both open and closed conformations on a rapid time scale. The closed form would not be able to interact productively with AztD. Thus, forcing AztC into the open form by deleting this loop would increase the rate of transfer. A direct measurement of dynamics of holo and apo AztC by NMR would be of interest, and these experiments are currently underway in our laboratory.

The presence of a Z-Loop would seem likely to confer an advantage by preventing the premature dissociation of zinc from the SBP. However, most organisms are able to survive zinc limitation with cluster A-ISBPs lacking a Z-Loop, making its physiological function and relevance uncertain. It may also function as a recognition element, allowing specific interaction with the membrane permease. We are currently working to generate Z-Loop deletion mutations in vivo to address this unresolved question.

\section{3. $H 138 / 204 A$ AztC}

Our initial intent was to generate a mutant AztC that was able to interact with AztD but not bind zinc. Thus, it could act as a competitive inhibitor of transfer to WT and allow us to estimate a binding affinity between the two proteins. The simplest means to that end seemed to be removing the ligands at the zinc binding site. While we failed to achieve this outcome, the results from this mutant were surprising and may provide some insight into zinc binding and transfer.

Somewhat surprisingly, H138/204A AztC still bound zinc with high affinity, at the limit of the MF-2 assay. However, the off-rate for zinc was very rapid, suggesting that $\mathrm{k}_{\text {on }}$ for zinc must be near the diffusion limit $\left(\sim 10^{8} \mathrm{M}^{-1} \times \mathrm{s}^{-1}\right)$ to maintain the observed high affinity. It seems reasonable to assume this is true of $\mathrm{WT}$ as well, which would require a Kd for zinc in the mid to low picomolar range $\left(\sim 10^{-11} \mathrm{M}\right)$. The affinity of most SBPs for zinc is reported in the low $\mathrm{nM}$ range [16,42-45]. However, some of these are only upper limit estimates due to limitations of the binding assays, as is the case for the MF-2 assay. Even using isothermal titration calorimetry (ITC), it can be challenging to accurately measure extremely high binding affinities. Thus, it is possible in many cases, and likely in the case of AztC, that the affinities of some cluster A-I SBPs for zinc are underestimated.

Certainly, the most puzzling result from this work is the observation that inclusion of H138/204A AztC into the WT transfer reaction significantly enhances the rate of transfer. While we can offer no definitive explanation at this time, it would seem to suggest a more complex binding equilibrium between $\mathrm{AztC}$ and $\mathrm{AztD}$ than previously imagined. It could be that AztC can bind at more than one site on $\mathrm{AztD}$, and that binding to the remote site enhances the rate of transfer. Similarly, some self-association of AztC could speed the process. Evaluation of these possibilities will require interaction assays that are independent of zinc transfer. Labeling AztC and AztD proteins with fluorophores capable of Förster resonance energy transfer (FRET) would seem an attractive strategy. Alternatively, NMR may prove useful in this capacity as well. In any case, continued study of AztC/AztD is likely to provide additional mechanistic insights into zinc transfer between proteins. 


\section{Conclusions}

Here we show the role of certain structural features of AztC and AztD in zinc binding and transfer. The NTM of AztD appears to have no role in zinc transfer to AztC but does impact zinc binding. The Z-Loop of AztC functions to lock down over the zinc binding site, decreasing the rate of zinc dissociation. It also appears to inhibit the formation of productive interactions with AztD for transfer. Finally, a double mutant of zinc ligands (H138/204A) of AztC is able to significantly increase the rate of zinc transfer from AztD to WT AztC through an as-yet unknown mechanism. Taken together, this work validates certain aspects of a previously determined interaction model [26] while providing new and interesting avenues of investigation into this unusual system.

Author Contributions: Conceptualization, methodology, validation, investigation, formal analysis, and writing-review and editing A.M. and E.T.Y.; resources, data curation, writing-original draft preparation, project administration, funding acquisition, E.T.Y. All authors have read and agreed to the published version of the manuscript.

Funding: This research was funded by National Institute of General Medical Science of the National Institutes of Health under award number 1R01GM122819-01A1.

Acknowledgments: We acknowledge the staff at the Berkeley Center for Structural Biology at Lawrence Berkeley National Laboratory. The Berkeley Center for Structural Biology is supported in part by the National Institutes of Health, National Institute of General Medical Sciences, and the Howard Hughes Medical Institute. The Advanced Light Source is supported by the Director, Office of Science, Office of Basic Energy Sciences, of the U.S. Department of Energy under Contract No. DE-AC02-05CH11231. The atomic coordinates and structure factors for $\Delta \mathrm{Z}$-loop AztC (6XPN) have been deposited in the Protein Data Bank, Research Collaboratory for Structural Bioinformatics, Rutgers University, New Brunswick, NJ (http://www.rcsb.org/).

Conflicts of Interest: The authors declare no conflict of interest. The funders had no role in the design of the study; in the collection, analyses, or interpretation of data; in the writing of the manuscript, or in the decision to publish the results.

\section{References}

1. Magistrato, A.; Pavlin, M.; Qasem, Z.; Ruthstein, S. Copper trafficking in eukaryotic systems: Current knowledge from experimental and computational efforts. Curr. Opin. Struct. Biol. 2019, 58, 26-33. [CrossRef] [PubMed]

2. Kambe, T.; Tsuji, T.; Hashimoto, A.; Itsumura, N. The Physiological, Biochemical, and Molecular Roles of Zinc Transporters in Zinc Homeostasis and Metabolism. Physiol. Rev. 2015, 95, 749-784. [CrossRef] [PubMed]

3. Blindauer, C.A. Bacterial metallothioneins: Past, present, and questions for the future. JBIC J. Biol. Inorg. Chem. 2011, 16, 1011-1024. [CrossRef]

4. Nairn, B.L.; Lonergan, Z.; Wang, J.; Braymer, J.; Zhang, Y.; Calcutt, M.W.; Lisher, J.P.; Gilston, B.A.; Chazin, W.J.; De Crécy-Lagard, V.; et al. The Response of Acinetobacter baumannii to Zinc Starvation. Cell Host Microbe 2016, 19, 826-836. [CrossRef] [PubMed]

5. Chandrangsu, P.; Huang, X.; Gaballa, A.; Helmann, J.D. Bacillus subtilis FolE is sustained by the ZagA zinc metallochaperone and the alarmone ZTP under conditions of zinc deficiency. Mol. Microbiol. 2019, 112, 751-765. [CrossRef]

6. Neupane, D.P.; Jacquez, B.; Sundararajan, A.; Ramaraj, T.; Schilkey, F.D.; Yukl, E.T. Zinc-Dependent Transcriptional Regulation in Paracoccus denitrificans. Front. Microbiol. 2017, 8. [CrossRef]

7. Biemans-Oldehinkel, E.; Doeven, M.K.; Poolman, B. ABC transporter architecture and regulatory roles of accessory domains. FEBS Lett. 2005, 580, 1023-1035. [CrossRef] [PubMed]

8. Higgins, C.F. ABC Transporters: From Microorganisms to Man. Annu. Rev. Cell Biol. 1992, 8, 67-113. [CrossRef] [PubMed]

9. Berntsson, R.P.-A.; Smits, S.H.J.; Schmitt, L.; Slotboom, D.-J.; Poolman, B. A structural classification of substrate-binding proteins. FEBS Lett. 2010, 584, 2606-2617. [CrossRef]

10. Loisel, E.; Jacquamet, L.; Serre, L.; Bauvois, C.; Ferrer, J.L.; Vernet, T.; Di Guilmi, A.M.; Durmort, C. AdcAII, A New Pneumococcal Zn-Binding Protein Homologous with ABC Transporters: Biochemical and Structural Analysis. J. Mol. Biol. 2008, 381, 594-606. [CrossRef] 
11. Hood, M.I.; Skaar, E.P. Nutritional immunity: Transition metals at the pathogen-host interface. Nat. Rev. Genet. 2012, 10, 525-537. [CrossRef] [PubMed]

12. Kehl-Fie, T.E.; Skaar, E.P. Nutritional immunity beyond iron: A role for manganese and zinc. Curr. Opin. Chem. Biol. 2010, 14, 218-224. [CrossRef] [PubMed]

13. Panina, E.M.; Mironov, A.A.; Gelfand, M.S. Comparative genomics of bacterial zinc regulons: Enhanced ion transport, pathogenesis, and rearrangement of ribosomal proteins. Proc. Natl. Acad. Sci. USA 2003, 100, 9912-9917. [CrossRef] [PubMed]

14. Plumptre, C.D.; Eijkelkamp, B.A.; Morey, J.R.; Behr, F.; Couñago, R.M.; Ogunniyi, A.D.; Kobe, B.; O'Mara, M.L.; Paton, J.C.; McDevitt, C.A. AdcA and AdcAII employ distinct zinc acquisition mechanisms and contribute additively to zinc homeostasis in Streptococcus pneumoniae. Mol. Microbiol. 2014, 91, 834-851. [CrossRef] [PubMed]

15. Cao, K.; Li, N.; Wang, H.; Cao, X.; He, J.; Zhang, B.; He, Q.-Y.; Zhang, G.; Sun, X. Two zinc-binding domains in the transporter AdcA from Streptococcus pyogenes facilitate high-affinity binding and fast transport of zinc. J. Biol. Chem. 2018, 293, 6075-6089. [CrossRef] [PubMed]

16. Ilari, A.; Alaleona, F.; Tria, G.; Petrarca, P.; Battistoni, A.; Zamparelli, C.; Verzili, D.; Falconi, M.; Chiancone, E. The Salmonella enterica ZinT structure, zinc affinity and interaction with the high-affinity uptake protein ZnuA provide insight into the management of periplasmic zinc. Biochim. Biophys. Acta 2014, 1840, 535-544. [CrossRef]

17. Petrarca, P.; Ammendola, S.; Pasquali, P.; Battistoni, A. The Zur-Regulated ZinT Protein Is an Auxiliary Component of the High-Affinity ZnuABC Zinc Transporter That Facilitates Metal Recruitment during Severe Zinc Shortage. J. Bacteriol. 2010, 192, 1553-1564. [CrossRef]

18. Gabbianelli, R.; Scotti, R.; Ammendola, S.; Petrarca, P.; Nicolini, L.; Battistoni, A. Role of ZnuABC and ZinT in Escherichia coli O157:H7 zinc acquisition and interaction with epithelial cells. BMC Microbiol. 2011, 11, 36. [CrossRef]

19. Graham, A.I.; Hunt, S.; Stokes, S.L.; Bramall, N.; Bunch, J.; Cox, A.G.; McLeod, C.W.; Poole, R.K.; Oka, M.; Sumita, N.; et al. Severe Zinc Depletion of Escherichia coli. J. Biol. Chem. 2009, 284, 18377-18389. [CrossRef]

20. Eijkelkamp, B.A.; Pederick, V.G.; Plumptre, C.D.; Harvey, R.M.; Hughes, C.E.; Paton, J.C.; McDevitt, C.A. The First Histidine Triad Motif of PhtD Is Critical for Zinc Homeostasis in Streptococcus pneumoniae. Infect. Immun. 2015, 84, 407-415. [CrossRef]

21. Ogunniyi, A.D.; Grabowicz, M.; Mahdi, L.K.; Cook, J.; Gordon, D.L.; Sadlon, T.A.; Paton, J.C. Pneumococcal histidine triad proteins are regulated by the Zn 2+-dependent repressor AdcR and inhibit complement deposition through the recruitment of complement factor H. FASEB J. 2008, 23, 731-738. [CrossRef] [PubMed]

22. Bersch, B.; Bougault, C.; Roux, L.; Favier, A.; Vernet, T.; Durmort, C. New Insights into Histidine Triad Proteins: Solution Structure of a Streptococcus pneumoniae PhtD Domain and Zinc Transfer to AdcAII. PLOS ONE 2013, 8, e81168. [CrossRef] [PubMed]

23. Handali, M.; Roychowdhury, H.; Neupane, D.P.; Yukl, E.T. AztD, a Periplasmic Zinc Metallochaperone to an ATP-binding Cassette (ABC) Transporter System in Paracoccus denitrificans. J. Biol. Chem. 2015, 290, 29984-29992. [CrossRef] [PubMed]

24. Handali, M.; Neupane, D.P.; Roychowdhury, H.; Yukl, E.T. Transcriptional Regulation, Metal Binding Properties and Structure of Pden1597, an Unusual Zinc Transport Protein from Paracoccus denitrificans. J. Biol. Chem. 2015, 290, 11878-11889. [CrossRef]

25. Neupane, D.P.; Avalos, D.; Fullam, S.; Roychowdhury, H.; Yukl, E.T. Mechanisms of zinc binding to the solute-binding protein AztC and transfer from the metallochaperone AztD. J. Biol. Chem. 2017, 292, 17496-17505. [CrossRef]

26. Neupane, D.P.; Fullam, S.H.; Chacón, K.N.; Yukl, E.T. Crystal structures of AztD provide mechanistic insights into direct zinc transfer between proteins. Commun. Biol. 2019, 2, 308-312. [CrossRef]

27. Edelhoch, H. Spectroscopic Determination of Tryptophan and Tyrosine in Proteins*. Biochemistry 1967, 6, 1948-1954. [CrossRef]

28. Golynskiy, M.V.; Gunderson, W.A.; Hendrich, M.P.; Cohen, S.M. Metal Binding Studies and EPR Spectroscopy of the Manganese Transport Regulator MntR. Biochemistry 2006, 45, 15359-15372. [CrossRef]

29. Kuzmic, P. Program DYNAFIT for the Analysis of Enzyme Kinetic Data: Application to HIV Proteinase. Anal. Biochem. 1996, 237, 260-273. [CrossRef]

30. Kuzmic, P. DynaFit-A Software Package for Enzymology. Methods Enzymol. 2009, 467, 247-280. [CrossRef] 
31. Kabsch, W. XDS. Acta Cryst. 2010, 66, 125-132. [CrossRef] [PubMed]

32. Kabsch, W. Integration, scaling, space-group assignment and post-refinement. Acta Cryst. 2010, 66, 133-144. [CrossRef] [PubMed]

33. McCoy, A.J.; Grosse-Kunstleve, R.W.; Adams, P.D.; Winn, M.D.; Storoni, L.C.; Read, R.J. Phaser crystallographic software. J. Appl. Crystallogr. 2007, 40, 658-674. [CrossRef] [PubMed]

34. Emsley, P.; Cowtan, K. Coot: Model-building tools for molecular graphics. Acta Cryst. 2004, 60, $2126-2132$. [CrossRef]

35. Adams, P.D.; Afonine, P.V.; Bunkóczi, G.; Chen, V.B.; Davis, I.W.; Echols, N.; Headd, J.J.; Hung, L.-W.; Kapral, G.J.; Grosse-Kunstleve, R.W.; et al. PHENIX: A comprehensive Python-based system for macromolecular structure solution. Acta Cryst. 2010, 66, 213-221. [CrossRef]

36. Zheng, B.; Zhang, Q.; Gao, J.; Han, H.; Li, M.; Zhang, J.; Qi, J.; Yan, J.; Gao, G.F. Insight into the Interaction of Metal Ions with TroA from Streptococcus suis. PLOS ONE 2011, 6, e19510. [CrossRef]

37. Lee, Y.H.; Deka, R.K.; Norgard, M.V.; Radolf, J.D.; A Hasemann, C. Treponema pallidum TroA is a periplasmic zinc-binding protein with a helical backbone. Nat. Genet. 1999, 6, 628-633. [CrossRef]

38. Ragunathan, P.; Spellerberg, B.; Ponnuraj, K. Structure of laminin-binding adhesin (Lmb) from Streptococcus agalactiae. Acta Cryst. 2009, 65, 1262-1269. [CrossRef]

39. Linke, C.; Caradoc-Davies, T.T.; Young, P.G.; Proft, T.; Baker, E.N. The Laminin-Binding Protein Lbp from Streptococcus pyogenes Is a Zinc Receptor. J. Bacteriol. 2009, 191, 5814-5823. [CrossRef]

40. Lee, Y.-H.; Dorwart, M.R.; Hazlett, K.R.O.; Deka, R.K.; Norgard, M.V.; Radolf, J.D.; Hasemann, C.A. The Crystal Structure of Zn(II)-Free Treponema pallidum TroA, a Periplasmic Metal-Binding Protein, Reveals a Closed Conformation. J. Bacteriol. 2002, 184, 2300-2304. [CrossRef]

41. Sridharan, U.; Ragunathan, P.; Spellerberg, B.; Ponnuraj, K. Molecular dynamics simulation of metal free structure of Lmb, a laminin-binding adhesin of Streptococcus agalactiae: Metal removal and its structural implications. J. Biomol. Struct. Dyn. 2018, 37, 714-725. [CrossRef] [PubMed]

42. Yatsunyk, L.A.; Easton, J.A.; Kim, L.R.; Sugarbaker, S.A.; Bennett, B.; Breece, R.M.; Vorontsov, I.I.; Tierney, D.L.; Crowder, M.W.; Rosenzweig, A.C. Structure and metal binding properties of ZnuA, a periplasmic zinc transporter from Escherichia coli. J. Biol. Inorg. Chem. 2007, 13, 271-288. [CrossRef] [PubMed]

43. Wei, B.; Randich, A.M.; Bhattacharyya-Pakrasi, M.; Pakrasi, H.B.; Smith, T.J. Possible Regulatory Role for the Histidine-Rich Loop in the Zinc Transport Protein, ZnuA. Biochemistry 2007, 46, 8734-8743. [CrossRef] [PubMed]

44. Desrosiers, D.C.; Sun, Y.C.; Zaidi, A.A.; Eggers, C.H.; Cox, D.L.; Radolf, J.D. The general transition metal (Tro) and $\mathrm{Zn} 2+(\mathrm{Znu})$ transporters in Treponema pallidum: Analysis of metal specificities and expression profiles. Mol. Microbiol. 2007, 65, 137-152. [CrossRef] [PubMed]

45. Abate, F.; Malito, E.; Cozzi, R.; Surdo, P.L.; Maione, M.; Bottomley, M.J. Apo, Zn2+-bound and Mn2+-bound structures reveal ligand-binding properties of SitA from the pathogen Staphylococcus pseudintermedius. Biosci. Rep. 2014, 34, 743-758. [CrossRef]

(C) 2020 by the authors. Licensee MDPI, Basel, Switzerland. This article is an open access article distributed under the terms and conditions of the Creative Commons Attribution (CC BY) license (http://creativecommons.org/licenses/by/4.0/). 